\title{
Novel Taste Facilitation of the Association of Visual Cues with Toxicosis in Rats
}

\author{
Bennett G. Galef, Jr. and Bob Osborne \\ McMaster University, Hamilton, Canada
}

\begin{abstract}
The present experiments examined the conditions under which rats rapidly learn to avoid ingesting visually distinctive food objects associated with toxicosis. It was found that the presence of a novel taste associated with a visually distinctive food object faciliated acquisition of visual-cue-toxicosis associations. Further experiments failed to support either higher order conditioning or sensory preconditioning models of this phenomenon. The results are discussed in terms of species' differences in the conditions under which attention is directed to visual cues associated with ingesta. The implications of these findings for the existence of visual aposomatisms (warning colors) in naturally occurring toxic species are also examined.
\end{abstract}

Many potential-prey species have evolved the capacity to synthesize or sequester substances capable of producing aversive physiological states in potential predators. Such toxins have been shown to play an important role in protecting species possessing them from predation (see, e.g., Brower, 1969; Brower, Ryerson, Coppinger, \& Glazier, 1968). Toxicity of species members is not, in itself, however, sufficient to provide protection from predation. In order for the toxicity of species members to result in inhibition of predation, potential predators must be able to associate stimulus characteristics of toxic species members with aversive postingestional events. ${ }^{1}$ Should potential predators be unable to learn such associations, toxic. prey would gain no protection from predation as a result of their toxicity. Predatory individuals would simply continue ingesting members of toxic species and suffering toxicosis.

It is clearly in the best interests of toxic-

The research reported here was supported by $\mathrm{Na}$ tional Research Council of Canada Grant AP307 and a McMaster University Research Grant to the first author and by National Research Council of Canada Grant APA0042 to A. H. Black. We thank Sue Johns, Jan Trafford, and Mark Hammer for their technical assistance and Sara Shettleworth and Marvin Krank for their thoughtful critiques of an earlier draft of the manuscript.

Requests for reprints should be sent to Bennett (i. Galef, Ir., Department of Psychology, McMaster University, Hamilton, Ontario l $8 \mathrm{~S} 4 \mathrm{~K} 1$, Canada. prey species to exhibit stimulus characteristics that are boith readily discriminable from those of benign sympatrics and easily associated by predators with the aversive events consequent upon ingestion of toxic individuals. In fact, many if not all noxious species have evolved distinctive, speciestypical morphological and/or behavioral phenotypes. Such aposomatisms generally take the form of bright colors, contrasting visual patterns, distinctive locomotor patterns, noises, or smells (Edmunds, 1974).

It has been widely held in the psychological literature (Garcia \& Ervin, 1968; Rozin \& Kalat, 1971; Seligman, 1970) that although rats, and by inference many other mammals, readily learn to associate gustatory or olfactory (interoceptive) cues with toxicosis, they find it difficult or impossible to associate visual or auditory (telereceptive) cues with aversive internal events. Although recent evidence (see, e.g., Best, Best, \& Mickley, 1973; Mitchell, Kirschbaum, \& Perry, 1975; Morrison \& Collyer, 1974) indicates that the formation of visual-cue-toxicosis associations is possible in rats, such demonstrations have required large numbers of training trials to produce reliable evidence of telereceptive-cue-toxicosis conditioning.

\footnotetext{
1 An alternative basis for the success of the toxicity aposomatism strategy as a defense against predators, innate avoidance of prey aposomatisms by predators (Smith, 1975), is not further discussed here, as it is not of immediate relevance.
} 
'This difficulty in establishing telereceptive-cue-toxicosis learning contrasts markedly with the robust effects produced by a single association of toxicosis with gustatory cues and can be considered evidence supportive of the view that interoceptive cues are far more easily associated with toxicosis than telereceptive ones.

The preceding discussion leads to two unlikely conclusions: first, that aposomatic visual patterns, sounds, and behavior, which are common in nature, are not very useful in protecting toxic prey from capture and sampling by predators whose learning capacities are similar to those of rats and, second, that such predators find it difficult to take advantage of many telereceptive aposomatic signals that would preclude their wasting energy pursuing, capturing, and tasting toxic prey. It seems to us far more probable, in an ecological context, that predators in general and rats in particular can, in fact, readily associate toxicosis with telereceptive cues but under conditions different from those usually prevailing in laboratory tests of the associability of telereceptive events with subsequent aversive internal states.

We consider the natural situation to be one in which a mammalian predator (a) approaches a telereceptively distinctive prey, (b) tastes the prey object and experiences an unpleasant gustatory sensation resulting from the taste of the toxin (many naturally occurring toxins, including alkaloids and cardiac glycosides, are bitter; Brower, 1969; Garcia \& Hankins, 1975), and (c) some time later experiences an aversive internal state as a result of the physiological action of the ingested toxin. It seemed possible to us that for many mammals the presence of an aversive taste in conjunction with telereceptive cues might be a sufficient condition for the formation of associations between those telereceptive cues and any aversive internal states following them.

The choice of a species in which to test the preceding hypothesis poses something of a problem. It might well be argued on ecological grounds that, given the rationale for the present studies, Rattus norvegicus would be a particularly inappropriate choice. Not only have we failed to find evidence in the literature that rats encounter aposomatic toxic prey in the wild, but in addition it is well established that rats feed most frequently in hours of darkness when visual cues are difficult to utilize. On the other hand, models of poison avoidance learning in the psychological literature most frequently treat the behavior of rats as representing the general mammalian case, from which, for example, one extrapolates to poison avoidance behavior of humans or that of coyotes. The taste aversion learning of other species is by contrast viewed as, to some extent, idiosyncratic and specialized.

Thus, the species chosen for the present work is necessarily undesirable from either the psychological or the ecological point of view. Because the work described below was more directly addressed to questions concerning the proximal causation of behavior than its function, we made the ecologically inappropriate choice of subject species.

The first experiment examines the acquisition of the avoidance of ingestion of visually distinctive objects associated with toxicosis by rats as a function of the presence of novel taste cues. Evidence that the presence of an aversive or other novel taste in association with a visual cue is a sufficient condition for the rapid learning of an association between that visual cue and toxicosis would provide a resolution of the apparent inconsistency between findings of telereceptive aposomatisms in nature and the difficulty that some mammals exhibit in forming associations between telereceptive cues and toxicosis.

\section{Experiment 1}

In the present experiment, rats were subjected to toxicosis following ingestion of visually distinctive objects of varying flavor to determine the sufficiency of novel bitter, novel sweet, and familiar flavors to facilitate the association of visual cues with toxicosis.

\section{Method}

Subjects. Sixty male Long-Evans rats obtained from the Canadian Breeding Farms, St. Constant, Quebec, weighing $175-200 \mathrm{~g}$, served as subjects. 
Table 1

Contents of Presented Capsules and Substances Injected on Each Day of Experiment 1

\begin{tabular}{|c|c|c|c|c|c|c|}
\hline \multirow[b]{2}{*}{ Group } & \multirow{2}{*}{$\begin{array}{l}\text { Pretraining } \\
\text { capsules } \\
\text { (Days } 1 \text { and } \\
\frac{2 \text { ) }}{\text { Clear }(10)}\end{array}$} & \multicolumn{2}{|c|}{$\begin{array}{c}\text { Training capsules } \\
\text { (Day 3) }\end{array}$} & \multirow{2}{*}{$\begin{array}{c}\text { Injection } \\
\text { (Day } 3 \text { ) }\end{array}$} & \multicolumn{2}{|c|}{$\begin{array}{l}\text { Testing capsules } \\
\text { (1)ays } 4 \text { and 5) }\end{array}$} \\
\hline & & Clear (8) & Distinctive (2) & & Clear $(8)$ & Distinctive (2) \\
\hline Bitter-Poison & Purina & Purina & $4 \%$ quinine & $.12 \mathrm{M} \mathrm{LiCl}$ & Purina & Purina \\
\hline Bitter & Purina & Purina & $4 \%$ quinine & saline & Purina & Purina \\
\hline Poison & Purina & Purina & Purina & $.12 \mathrm{M} \mathrm{LiCl}$ & Purina & Purina \\
\hline Control & Purina & Purina & Purina & saline & Purina & Purina \\
\hline Sweet-Poison & Purina & Purina & $50 \%$ sucrose & $.12 \mathrm{M} \mathrm{LiCl}$ & Purina & Purina \\
\hline Sweet & Purina & Purina & $50 \%$ sucrose & saline & Purina & Purina \\
\hline
\end{tabular}

Note. The numbers in the parentheses are the numbers of capsules presented.

Procedure. For 3 wk prior to the initiation of experimental procedures and for the five subsequent days of pretraining, training, and testing, each subject was housed individually, handled daily, and maintained on ad lib water and a $3 \mathrm{hr} /$ day feeding schedule (powdered Purina Laboratory Chow, 1:30-4:30 p.m.). Subjects were individually pretrained, trained, and tested between 9 a.m. and 1 p.m. in a sound-attenuating room with background white noise.

Apparatus. Individual subjects were exposed to experimental procedures in a $40 \times 40 \times 40 \mathrm{~cm}$ wooden enclosure. A $5 \times 5 \mathrm{~cm}$ opening in one wall permitted access to the enclosure from a $23 \times 11 \times 16 \mathrm{~cm}$ start box with a removable guillotine door. Food items were presented on a 2 -cm-high, 2 -cm-wide platform with 10 shallow slots $2.5 \mathrm{~cm}$ center to center located against the enclosure wall opposite the guillotine door. Both start box and enclosure had clear Plexiglas lids to permit observation of the individual subject's behavior.

Procedure. Pretraining (Days 1 and 2): Each subject was pretrained to feed in the apparatus for two consecutive days. On each pretraining day a No. 2 clear gelatin capsule (Parke-Davis) filled with a mean of .31 $\mathrm{g}$ of powdered Purina Laboratory Chow was placed in each of the 10 slots in the feeding platform. The subject was then removed from its home cage, weighed, and placed in the start box. The guillotine door was removed, and the subject was left undisturbed in the apparatus until either it had ingested all 10 capsules or 55 $\mathrm{min}$ had elapsed. Any subject failing to ingest all 10 capsules on either pretraining day within the $55 \mathrm{~min}$ allowed was dropped from the experiment.

Training (Day 3): Training was initiated $21 \mathrm{hr}$ subsequent to completion of the second day of pretraining. Fach subject was randomly assigned to one of the six groups the treatment of which is described in Table 1. On the training day each subject was presented with eight clear No. 2 capsules, each containing .31 $\mathrm{g}$ of powdered Purina laboratory Chow, and with two distinctive No. 2 capsules (half clear gelatin and half blue gelatin. Examination of the distinctive and plain capsules on closed-circuit black-and-white television revealed, that, to the human eye at least, they were readily discriminable even in the absence of color vision). The contents of these two distinctive capsules varied among groups. Both of the distinctive capsules presented daily to each animal contained, as indicated in Table 1 , unadulterated Purina chow, Purina chow adulterated with $4 \%$ by weight quinine hydrochloride, or Purina chow adulterated with $50 \%$ by weight brown sugar.

Ten minutes after each subject had initiated ingestion of its first distinctive capsule, it was removed from the apparatus and injected, according to group assignment, with either $2 \%$ of body weight of $.12 \mathrm{M}$ lithium chloride or of saline solution, as indicated in Table 1. Following injection, the subject was returned to its home cage.

Testing (Days 4 and 5): On each of the two subsequent days, each subject was again presented with ejght clear and two distinctive capsules. However, on testing days all 10 capsules contained unadulterated powdered Purina Laboratory Chow. Each test session was terminated when a subject had completed ingestion of all 10 capsules or 8 min had elapsed without the subject's feeding.

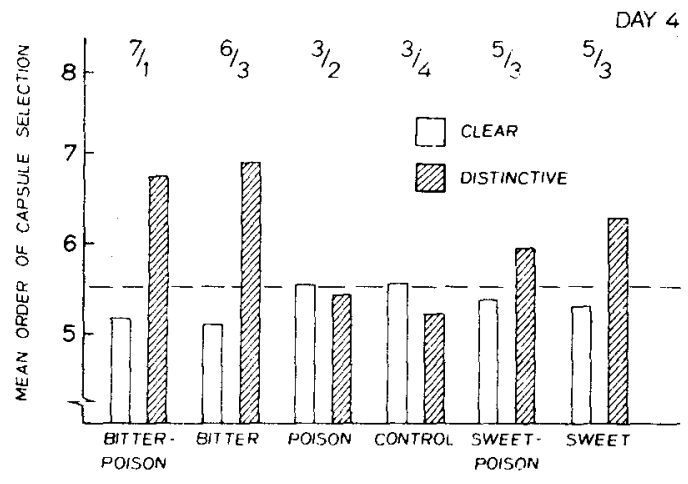

Figure 1. Mean order of selection of distinctive and clear capsules from food slots on Days 4 and 5 for the six groups the treatment of which is described in 'Table 1. ('The numerator of each fraction indieates the number of subjects in a group exhibiling a higher mean order of selection of distinctive apsules than clear ones; the denominator indicates the number of subjects exhibiting a lower mean order of selection of distinctive capsules than clear ones. 'The dotted line represents the expected mean order of selection.) 




Figure 2. Mean latency to complete ingestion of distinctive and clear capsules on Days 4 and 5 . (The flags indicate $\pm 1 S . E$.

On each day of testing, the observer recorded the order of removal of capsules from the food presentation slots and the time to termination of ingestion of each capsule. Conditions were arranged so that the observer did not know the group assignment of individual subjects until the experiment was completed.

\section{Results}

Although all the dependent measures of food selection and ingestion in Experiment 1 pointed to the conclusions described below, determination of the order of removal of capsules from the slots in the feeding platform posed greater difficulties than we had anticipated. Rats frequently both accidentally dislodged capsules from slots and moved capsules from place to place in the apparatus without initiating ingestion. The variability introduced into the measure of order of capsule selection by these behaviors considerably reduced our confidence in its meaningfulness, and we therefore placed rreater emphasis on measures of time to completion of ingestion of capsules in our description and interpretation of results.
The main results of Experiment 1 are presented in Figures 1, 2, and 3 which show, respectively, (a) the mean order of removal of distinctive and clear capsules from food slots on Day 4 by subjects in the six treatment groups described in Table 1 , (b) the mean latency to completion of ingestion of clear and distinctive capsules by subjects in each group on Days 4 and 5, and (c) the percentage of subjects in each of the six groups whose mean latency to ingest distinctive capsules was more than 1 or more than $2 S D$ greater than their mean latency to ingest clear capsules.

As is clear from examination of the figures, and as statistical analyses confirm, subjects in the Bitter-Poison group (a) exhibited greater hesitancy in selecting distinctive than clear capsules from the slots on Day 4 (Wilcoxon test, $T=8, p<.05$ ), (b) exhibited longer mean latencies to ingest distinctive than clear capsules on both Days 4 and 5 (Wilcoxon test, J)ay $4, T=0, p<.001$; D)ay $5, T^{\prime}=0, p<.(601)$, and (c) exhibited longer mean latencies to ingest distinctive capsules 




Figure 3. Percentage of subjects in each group whose mean latency to ingest distinctive capsules was more than 1 or more than $2 S D$ greater than their mean latency to ingest clear capsules.

on Day 4 than did subjects in the Bitter or Poison groups: Median test, Day $4, \chi^{2}(1)=$ 8.1, $p<.02$; Day $5, \chi^{2}(1)=2.6, .4<p<.2$.

The only other subjects exhibiting reliably longer latencies to ingest distinctive capsules than clear ones were those in the SweetPoison and Bitter groups on Day 4 (SweetPoison group, Wilcoxon test, $T=5, p<.02$; Bitter group, Wilcoxon test, $T=3, p<.005$ ). Subjects in the Control, Sweet, and Poison groups failed to exhibit reliably greater latencies to ingest distinctive than clear capsules on either Day 4 or Day 5. The failure of subjects in the Poison group to avoid distinctive capsules on Days 4 and 5 indicates that no aversion was learned to the taste of blue gelatin capsules.

The results of the present experiment suggest that rats do not, in fact, have great difficulty in associating distinctive visual features of food objects with toxicosis. To the contrary, they readily make such associations in the presence of a facilitating novel taste. Although the presence of a novel sweet taste is sufficient to support visualcue toxicosis associations, the facilitating effects of sweet taste are both less roloust and less longlasting than the facilitating effects of bitter taste.

\section{Discussion}

The facilitation of associations between visual cues and toxicosis in the Sweet-Poison and Bitter-Poison groups of the present experiment can be viewed as the result of one of two types of mediating process, either taste-mediated higher order conditioning or taste-mediated sensory preconditioning. In a recent article Garcia and Hankins (1977) discussed an earlier demonstration of aversive taste facilitation of visual-cue-toxicosis conditioning in Buteo hawks (Brett, Hankins, \& Garcia, 1976) as an instance of higher order conditioning, though the evidence on this point is not conclusive. The consummatory behavior of Brett et al.'s subjects was such that it allowed them to experience taste cues, visual cues, and toxicosis simultaneously, which makes difficult the analysis of mechanisms underlying the taste facilitation of the association of visual cues with toxicosis.

If a higher order conditioning model of the outcome of Experiment $I$ is appropriate, one might expect that subjects pretrained to associate a distinctive flavor with toxicosis and then given experience of distinctive visual cues in association with that taste would 
form robust aversions to the visual cues. The second experiment explores this possibility.

\section{Experiment 2}

If the novel taste facilitation of visualcue-toxicosis association demonstrated in Experiment 1 is the result of processes formally similar to higher order conditioning, then one would expect rats first trained to associate a novel taste $\left(\mathrm{CS}_{1}\right)$ with toxicosis (US) and subsequently trained to associate that novel taste $\left(\mathrm{CS}_{1}\right)$ with visually distinctive ingesta $\left(\mathrm{CS}_{2}\right)$, to thereafter exhibit a profound avoidance of similar visually distinctive ingesta. Because bitter taste was found in Experiment 1 to provide strong facilitation of visual-cue-toxicosis associations, it was decided to again use a bitter flavor to facilitate visual-cue-toxicosis learning. Evidence from Experiment 1 also indicated that bitter flavor in itself is sufficient to produce a significant avoidance of ingestion of visual cues with which it has been associated. The experimental question thus becomes whether pretraining of a bitter-taste-toxicosis association will strengthen the aversion to visual cues associated with a bitter taste. The Bitter-Poison, Bitter, and Poison groups of Experiment 1 were replicated to reaffirm the original finding, and a Conditioning-Control group was added to control for the effects of preexposure to bitter taste and toxicosis on the association of visual cues with bitter taste.

\section{Method}

Subjects. Fifty male Long-Evans rats obtained from the Canadian Breeding Farms, weighing 175-200 g, served as subjects.

l'rocedure'. 'The procedure employed with subjects in the Bitter, Poison, and Bitter-Poison groups of the present experiment was identical to that described in Method of Experiment 1.

Subjects in the Conditioning and ConditioningControl groups were treated identically to those in the Bitter group of Experiment 1 except on the 2 days immediately preceding the first day of pretraining, which are referred to below as l)ays -1 and -2 .

On the morribits of $\left[b_{i y}-1\right.$, each subject in the Conditioning group was presented in its home cage with a bowl containing powdered Purina Laboratory Chow adulterated with $4 \%$ by weight quinine hydrochloride.
Ten minutes following observation of clear rejection responses (cessation of feeding, face grooming, tooth grinding, etc), each subject was injected ip with $2 \%$ of body weight. $12 \mathrm{M} \mathrm{LiCl}$. Subjects in the Conditioning Control group were injected with $2 \%$ of body weight .12 $\mathrm{M} \mathrm{LiCl}$ on the morning of Day -2 and were presented with quinine-adulterated chow on Day -1 until they were observed to exhibit the clear rejection responses described above.

\section{Results and Discussion}

The main results of Experiment 2 are presented in Figures 4 and 5, which show, respectively, the mean latency to complete ingestion of capsules exhibited by subjects in the five groups of Experiment 2 on the first day of testing, and the number of subjects in each group whose mean latency to ingest distinctive capsules was more than 1 or more than $2 S D$ greater than their mean latency to ingest clear capsules on 'Test Days 4 and 5.

As is clear from comparison of the data describing the behavior of subjects in the Bitter-Poison, Poison, and Bitter groups of Experiment 2 with those of subjects in comparable groups in Experiment 1, the results of Experiment 1 were essentially replicated. Subjects in the Bitter-Poison and Bitter groups exhibited significantly longer latencies to ingest distinctive than clear capsules, and subjects in the Bitter-



Figure 4. Mean latency to complete ingestion of distinctive and clear capsules on Day 4 by subjects in Fxperiment 2. (Flags indicate \pm 1 S.E. Condit. = conditioning.) 


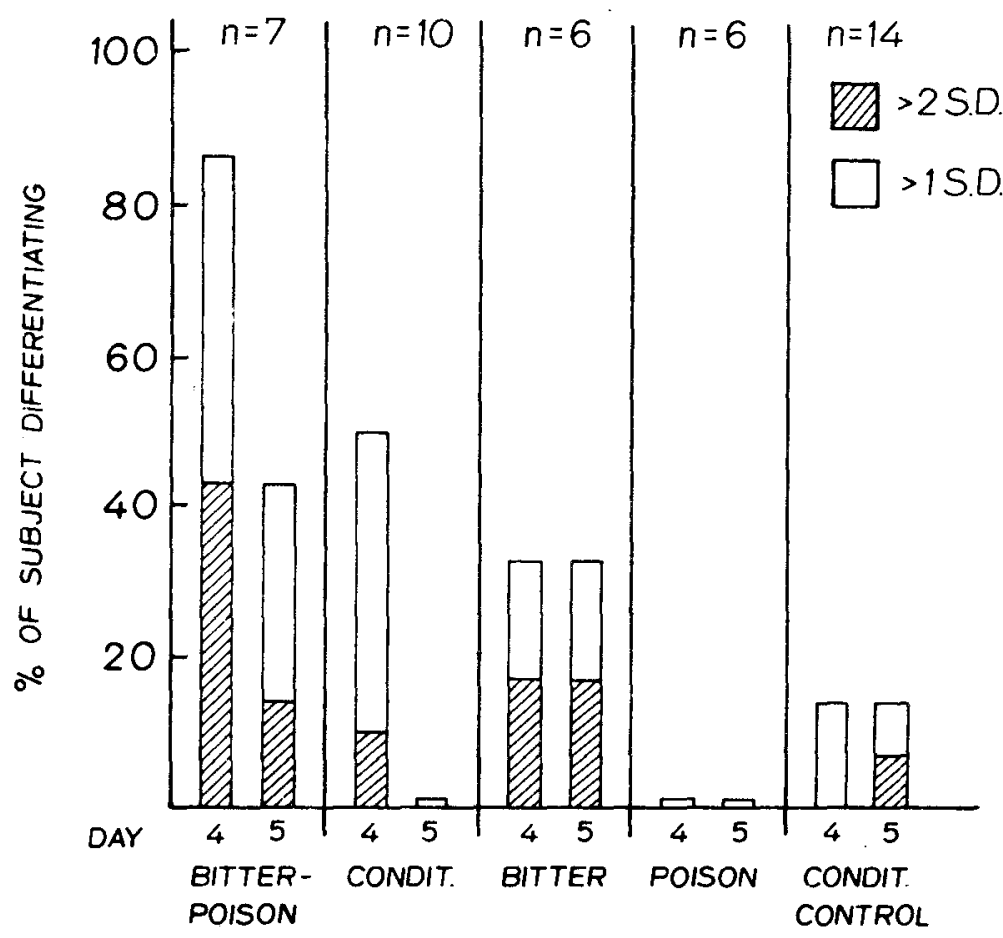

Figure 5. Percentage of subjects in each group of Experiment 2 whose mean latency to ingest distinctive capsules was more than 1 or more than $2 S D$ greater than their mean latency to ingest clear capsules. (Condit. = conditioning.)

Poison group exhibited far greater differentiation of clear and distinctive capsules than did subjects in the Bitter group.

Subjects in the Conditioning group did not exhibit greater differentiation than those in the Bitter group and, in fact, did not exhibit a significant differentiation in latencies to ingest clear and distinctive capsules (Wilcoxon test, $T=11, p>: 05$ ). These data fail to support a higher order conditioning explanation of the facilitating effects of novel tastes on visual-cue-toxicosis association in rats.

\section{Experiment 3}

Perhaps the most interesting feature of taste aversion learning is the ability of animals to tolerate long delays between exposure to gustatory cues and the onset of toxicosis and still form an association between those gustatory cues and toxicosis. The results of previous studies suggest that rats do not tolerate comparable delays between ingestion of novel visual cues and the onset of toxicosis. In particular, Wilcoxon, Dragoin, and Kral (1971), in an experiment formally similar in design to Experiment 1, found no evidence of visual-cue-toxicosis associations in rats exposed to a novel sourblue solution and poisoned $.5 \mathrm{hr}$ later. There is also preliminary evidence that even in the absence of mediating taste cues, rats form an aversion to visual cues associated with toxicosis if toxicosis occurs while ingestion of visually distinctive material is in progress (Braveman, 1977). The procedures of Experiment 1 resulted in the interval between ingestion of visually distinct material and toxicosis intermediate between the values present in the Braveman and the Wilcoxon et al. studies. It seemed possible, given the robust associations between visual cues and toxicosis resulting from a tastefacilitated single pairing with moderate conditioned stimulus unconditioned stimulus intervals found in Experiment 1, that rats in our paradigm might exhibit associa- 
tions between visual cues and toxicosis even if there was a considerable delay between presentation of the taste-mediated visual cue and toxicosis. The present experiment examines this possibility.

If sensory preconditioning is the mechanism underlying taste facilitation of the visual-cue-toxicosis associations demonstrated in the Sweet-Poison and BitterPoison groups of Experiments 1 and 2, one would expect taste-facilitated visual-cuetoxicosis associations with long delays between the visual cue and toxicosis so long as the visual and taste cues were experienced simultaneously and the delay between taste-cue presentation and toxicosis was not so great as to prohibit taste-toxicosis aversions from forming.

\section{Method}

Subjects. Subjects were eight male Long-Evans rats obtained from the Canadian Breeding Farms, weighing 175-200 g.

Procedure. The procedure was identical to that employed with the Bitter-Poison group of Experiment 1 except that injections of $\mathrm{LiCl}$ on Day 3 were not given until $1 \mathrm{hr}$ following initiation of ingestion of the first distinctive capsule. Each subject was returned to its home cage during the interval between removal from the test apparatus, 10 min after ingestion of the first distinctive capsule, and injection $50 \mathrm{~min}$ later.

\section{Results and Discussion}

The main results of Experiment 3 are presented in Figure 6A and 6B which show, respectively, (a) the mean latency to completion of ingestion of clear and distinctive capsules on Days 4 and 5 and (b) the percentage of subjects whose mean latency to ingest distinctive capsules was more than 1 $S D$ greater than their mean latency to ingest clear capsules. As is clear from examination of the figure, a 1-hr delay between ingestion of bitter distinctive capsules and toxicosis prevented the learning of a discriminated avoidance of those capsules.

The finding that rats do not form an aversion to visual cues associated with a bitter taste and followed $1 \mathrm{hr}$ later by toxicosis casts further doubt on the adequacy of mediational hypotheses to explain the role of novel tastes in the facilitation of the association of visual cues and toxicosis.
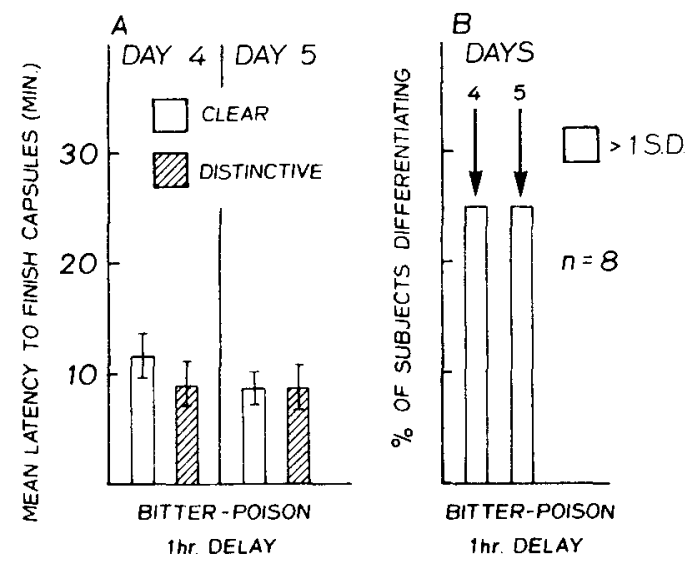

Figure 6. A: Mean latency to complete ingestion of distinctive and clear capsules on Days 4 and 5 by subjects in Experiment 3. B: Percentage of subjects in Experiment 3 whose mean latency to ingest distinctive capsules was more than $1 S D$ greater than their mean latency to ingest clear capsules.

Subjects in the present experiment experienced the visual and taste cues associated with the distinctive ingesta simultaneously and then experienced toxicosis after a $1 \mathrm{hr}$ delay. It is already well established in the literature that rats readily learn to associate novel taste cues and toxicosis separated by comparable intervals. Thus, the temporal relations between visual cue $\left(\mathrm{CS}_{1}\right)$ and taste $\left(\mathrm{CS}_{2}\right)$ and between taste $\left(\mathrm{CS}_{2}\right)$ and poison (US) should have been adequate to support learning by sensory preconditioning. The fact that such sensory preconditioning did not occur-although, of course, it is not sufficient grounds for rejecting sensory preconditioning explanations of the behavior of Bitter-Poison subjects in Experiments 1 and 2 -renders such interpretations less likely.

\section{General Discussion}

The results of the present experiments indicate that rats learn to associate visual cues with toxicosis if those visual cues are experienced in conjunction with a novel taste and are followed fairly rapidly by toxicosis onset. Our data fail to provide support for hypotheses suggesting that either higher order conditioning or sensory precondi- 
tioning provides the mechanism by which taste cues associated with ingesta facilitate the formation of associations between visual cues associated with those ingesta and toxicosis.

In retrospect it is, perhaps, not surprising to find circumstances in which rats readily learn to associate visual cues with toxicosis. A wide variety of other vertebrate species, e.g., quail (Wilcoxon et al., 1971), chickens (Capretta \& Moore, 1970), guinea pigs (Braveman, 1974, 1975), and codfish (MacKay, 1977), have already been found to do so.

Our data, taken together with other reports of visual-cue-toxicosis association in vertebrates, suggest that the major difference among species may lie not in their capacity to form associations between telereceptive cues and internal aversive events but rather in the conditions under which they attend to the visual cues associated with ingesta. In particular, we suggest that rats can directly associate visual cues with toxicosis but that they attend to the visual cues associated with ingesta only when those ingesta have a novel flavor. According to this model, rats in the Sweet-Poison group of Experiment 1, for example, would taste a distinctive capsule, discover it was sweet, acquire and store the information that they had ingested a capsule possessing distinctive visual features, and upon becoming ill, directly associate illness with ingestion of the distinctive visual cues. Subjects in the Poison group, lacking exposure to a novel taste at the time of ingestion of the visually distinctive capsules, would fail either to notice or to store the fact that they had ingested visually distinctive objects and, therefore, would not be able to form an aversion to those distinctive visual features when they subsequently experienced toxicosis. This model of taste-facilitated visual-cue-toxicosis conditioning is in some ways similar to prevailing views of gustatory-toxicosis association acquisition. In both cases the presence of a novel taste is seen as facilitating the association of foodrelated cues with toxicosis.

Such an interpretation of the data is consistent with a modified version of the "belongingness principle" proposed by Rozin and Kalat (1971; Rozin, 1977). We suggest, as have many others, that organisms tend to associate aversive internal events with food-related cues. We further suggest that the particular food-related cues to which subjects attend vary as a function both of species and of the particular stimulus complex characterizing the ingesta themselves. On this hypothesis, bobwhite quail are assumed to attend to visual cues present in food objects regardless of whether a novel taste is present in those food objects (Wilcoxon et al., 1971), guinea pigs are assumed to attend to visual cues present in food objects only when a salient taste cue is not associated with those food objects (Braveman, 1975), and rats are assumed to attend to visual cues present in food objects only when those food objects have a novel taste (particularly an unpalatable one) or are experienced in contiguity with internal malaise (Braveman, 1977). Thus, emphasis is placed on hypothesized differences in unconditioned attentional responses to the visual and gustatory aspects of ingesta rather than on hypothesized species differences in the capacity to form an association between visual cues and toxicosis.

Regardless of the mechanism proposed to account for the facilitative effects of novel tastes on the acquisition of avoidance responses to visual cues associated with toxicosis by rats, the outcome of Experiments 1 and 2 clearly resolve the contradiction explicated in the introduction of the present article. Visually aposomatic prey species having a bitter taste are protected from predation by organisms with learning capacities similar to those of rats. Visually aposomatic prey species that are bitter in taste and sequester toxins producing fairly rapid toxicosis onset are even better protected from attack by such predators. ${ }^{2}$

\footnotetext{
2 Some months before publication of this article, we received a manuscript by $\mathrm{K}$. W. Rusiniak, J. C. Hankins, J. Garcia, and L. P. Brett, demonstrating potentiation of odor-toxicosis conditioning by taste in rats and in a personal communication with $\mathrm{K}$. W. Rusiniak a brief description of a preprint by .J. C. C.lark, J. Irwin, and F. Westlorook, demonstrating potentiation of visualcue koxicosis association by taste in the homing pigeon. (Both manuseripls are in press in Bethomerel Biole(S.).
} 


\section{References}

Best, P. J., Best, M. R., \& Mickley, (. A. A. Conditioned aversion to distinct environmental stimuli resulting from gastro-intestinal distress. Journal of Comparative and Physiological Psychology, 1973, 82, $250-257$.

Braveman, N. S. Poison-based avoidance learning with flavored or colored water in guinea pigs. Learning and Motivation, 1974, 5, 182-194.

Braveman, N. S. Relative salience of gustatory and visual cues in the formation of poison based food inversions by guinea pigs (Cavia porcellus). Behavioral Biology, 1975, 14, 189-199.

Braveman, N. S. Visually guided avoidance of poisonous food in mammals. In L. M. Barker, M. R. Best, \& M. Domjan (Eds.), Learning mechanisms in food selection. Waco, Tex.: Baylor University Press, 1977.

Brett, L. P., Hankins, W. G., \& Garcia, J. Prey-lithium aversions: III: Buteo hawks. Behavioral Biology, $1976,17,87-98$.

Brower, L. P. Ecological chemistry. Scientific American, 1969, 220, 22-29.

Brower, L. P., Ryerson, W. N., Coppinger, L. L., \& Glazier, S. C. Ecological chemistry and the palatability spectrum. Science, 1968, 161, 1349-1350.

Capretta, P. J., \& Moore, M. Appropriateness of reinforcement to cue in the conditioning of food aversions in chickens (Gallus gallus). Journal of Comparative and Physiological Psychology, 1970, 72, 85-89.

Edmunds, M. Defense in animals. New York: Longman, 1974.

Garcia, J., \& Ervin, F. R. Gustatory-visceral and telereceptor-cutaneous conditioning: Adaptation in internal and external milieus. Communications in Behavioral Biology, 1968, 1, 389-415.

Garcia, J., \& Hankins, W. G. The evolution of bitter and the acquisition of toxiphobia. In D. A. Denton
\& J. P. Coghlan (Fds.), Olfaction and Tasto V. New York: Academic: Press, 1975.

Garcia, J., \& Hankins, W. (A. On the origin of food aversion paradigms. In L. M. Barker, M. R. Best, \& M. Domjan (Eds.), Learning mechanisms in food selection. Waco, Tex.: Baylor University Press, 1977.

MacKay, B. Visual and flavor cues in toxicosis conditioning of codfish. Behavioral Biology, 1977, 19, 87-97.

Mitchell, D., Kirschbaum, E. H., \& Perry, R. L. Effects of neophobia and habituation on the poison-induced avoidance of exteroceptive stimuli in the rat. Journal of Experimental Psychology: Animal Behavior Processes, 1975, 104, 47-55.

Morrison, G. R., \& Collyer, R. Taste-mediated conditioned aversion to an exteroceptive stimulus following LiCl poisoning. Journal of Comparative and Physiological Psychology, 1974, 86, 51-55.

Rozin, $P$. The significance of learning mechanisms in food selection: Some biology, psychology, and sociology of science. In I. M. Barker, M. R. Best, \& M. Domjan (Eds.), Learning mechanisms in ford selection. Waco, 'Tex.: Baylor University Press, 1977.

Rozin, P., \& Kalat, J. W. Specific hungers and poison avoidance as adaptive specializations of learning. Psychological Review, 1971, 78, 459-486.

Seligman, M. E. P. On the generality of the laws of learning. Psychological Review, 1970, 77, 400418.

Smith, S. Innate recognition of coral snake pattern by a possible avian predator. Science, 1975, 187, 759-760.

Wilcoxon, H. C., Dragoin, W. B., \& Kral, P. A. Illness induced aversions in rat and quail: Relative salience of visual and gustatory cues. Science, 1971, 171, $826-828$

Received October 18, 1977 